\title{
Knowledge and Adherence to Treatments for Genitourinary Syndrome Of Menopause in Covid-19 Pandemic: Comem-Gsm Study
}

Laura Baquedano Mainar ( $\sim$ lbaquedanome@hotmail.com )

Hospital Universitario Miguel Servet

Sonia Sanchez Mendez

Hospital General de Catalunya

Peña Dieste Pérez

Hospital Universitario Miguel Servet

Monica Hernandez Aragon

Hospital Universitario Miguel Servet

Nicolás Mendoza

University of Granada

\section{Research Article}

Keywords: Genitourinary syndrome of menopause, knowledge, menopause, COVID-19, confinement, adherence

Posted Date: June 15th, 2021

DOI: https://doi.org/10.21203/rs.3.rs-581688/v1

License: (c) (1) This work is licensed under a Creative Commons Attribution 4.0 International License. Read Full License 


\section{Abstract}

\section{Objective}

To study knowledge regarding genitourinary syndrome of menopause (GSM) and the treatments for it and to analyze treatment adherence during the COVID-19 confinement.

\section{Methods}

Multi-center observational study including women between 35 and 75 years. An extension study of treatment adherence was conducted during the coronavirus pandemic between March and April 2020

\section{Results}

A sample of 2355 women were included. Vaginal dryness was the most frequently identified symptom (74.3\%). Lubricants were the best-known treatments (69.6\%), followed by local estrogens $(25.7 \%) ; 66 \%$ of the women did not speak to their gynecologist about sexuality. Comparative analyses were conducted according to age, menopausal status, type of menopause, place of residence, type of health care received and level of education. During the coronavirus confinement period, adherence to treatments for vulvovaginal atrophy was poor in $72.5 \%$ asked $(n=204)$. Reduced sexual activity $(p>0.001)$ and coronavirus diagnosis $(p=0.003)$ were significantly associated with poorer treatment compliance.

\section{Conclusions}

There is great lack of knowledge of the treatments used for GSM. Most women do not talk to their gynecologist about sexuality. Adherence to treatments during the coronavirus confinement has been worryingly low.

\section{Background}

Genitourinary syndrome of menopause (GSM) is one of the most frequent complaints referred in transition and postmenopause and it could impact on the quality of life and sexual function women (1). Although sexuality is an important element of the lives of many midlife women (2), only a very small percentage of midlife women report having discussed their sexual problems with a healthcare professional (3).

If women are aware of the genital changes produced by hormonal decline and how this can affect their sexuality, it would be easier to identify women with dysfunction or possible symptoms that require treatment. There is an increasing tendency among clinicians to share the treatment options for vulvovaginal atrophy with the patient. The term "empowered therapy" has even been introduced in some publications (4-6). To make an informed choice, it is important that women have good knowledge about GSM that is free of myths and false beliefs. 
The aim of our study was to determine what women know about GSM. This study is part of a population survey known as the COMEM ("COnocimiento de las Mujeres Españolas en Menopausia" in Spanish) study. Data related to menopausal treatments have already been partially published elsewhere (7). As an extension to the latter study on menopause hormone therapy (MHT), in a sample of Spanish women, we aimed to evaluate the adherence to treatment for GSM during the period of confinement that was imposed due to the coronavirus pandemic (COVID-19).

\section{Methods}

This was a multi-center cross-sectional observational study carried out between January and June 2019, using a sample of 2500 women aged between 35 and 75 years. They participants were asked to complete an anonymous and voluntary survey written in simple and clear language.

A team of seven menopause experts selected questions structured in several clinical domains. The study related to therapies in menopause and socio-demographic characteristics of the sample the have been published previously (7). In the present study, we included the same cohort of women $(n=2355)$.

The questionnaire was offered to all women in the gynecology consulting-rooms of the participating centers, either they were patients or companions. Women who with physical, psychological or language incapacity to understand the survey questions, or those who did not want to participate were excluded from the study.

In the extension phase of the adherence study, women with treatment for GSM who had done the survey previously, were asked about the adherence and attitude to treatment during the state of alarm for coronavirus infection in Spain (from March 14 to June 21, 2020). Women who discontinued treatment before that time were not included in the extension phase. They were contacted by phone at the control visit after indicating treatment for GSM. Epidemiological data, sexual activity during confinement, type of treatment, dosage, and coronavirus infection data were collected. We evaluated adherence to pharmacological treatment using the Morisky-Green-Levine questionnaire with four items: each item has a dichotomous answer: 'yes' or 'no'. The questions ask if the patient takes the medicine correctly, if she has forgotten and if she abandons it in certain circumstances. The total score ranges from 0 to 4 points. A score of 4 points indicates good adherence to treatment, whilst less than 4 points indicates poor adherence (8).

The research was conducted in accordance with Good Clinical Practice standards and the current revision of the Declaration of Helsinki. The study was approved by the Research Ethics Committee of Aragon Spain (C.I. PI18/374) and of each involved center and was included in the Spanish Menopause Society National Research Network (REIM).

\section{Statistical analysis}


Categorical variables were reported in frequencies and percentages. Quantitative variables were showed using mean and standard deviation. Chi-Square test / Fisher's exact test or Student's T-test were used for each question of the questionnaire. The Mann-Whitney $U$ test was used for the analysis of dichotomous variables and the Kruskal-Wallis test for non-dichotomous variables when the distribution was nonparametric. The statistically significance was considered as $p$ value $<0.05$.

Data was collected and codified using IBM Statistics Process Social Sciences 22.0 for Windows (Copyright@ SPSS Inc., 2013) for subsequent statistical analysis.

\section{Results}

The answers obtained to the questions of the questionnaire are shown in Table 1. Vaginal dryness was a symptom identified with menopause by $74.3 \%$ of the women surveyed and decreased sexual desire by $44.8 \%$. Sexual dysfunction was recognized as a disease associated with menopause by $29 \%$. When asked how menopause affects sexuality, $65 \%$ answered that menopause decreases sexual desire. Pain with sexual activity and decreased frequency of sexual intercourse were identify by $34.9 \%$ and $27.4 \%$ of women asked respectively. Urinary incontinence was associated with menopause by $54 \%$ of the women surveyed, while urinary infections in $10.6 \%$. 
Table 1

GSM knowledge survey and responses given by women surveyed

\begin{tabular}{|c|c|c|}
\hline & $\mathrm{n}$ & $\%$ of total respondents $(n=2355)$ \\
\hline \multicolumn{3}{|c|}{ Which symptoms are associated with menopause? } \\
\hline None & 14 & $.6 \%$ \\
\hline Decreased sexual desire & 1054 & $44.8 \%$ \\
\hline Vaginal dryness & 1749 & $74.3 \%$ \\
\hline Pain during intercourse & 822 & $34.9 \%$ \\
\hline Vaginal itchiness & 298 & $12.6 \%$ \\
\hline Vulvar itchiness & 876 & $37.2 \%$ \\
\hline $\mathrm{DK}^{\star}$ & 28 & $1.2 \%$ \\
\hline \multicolumn{3}{|c|}{ Which conditions are associated with menopause? } \\
\hline None & 99 & $4.2 \%$ \\
\hline Sexual dysfunction & 682 & $29.0 \%$ \\
\hline Vaginal atrophy & 1766 & $75.0 \%$ \\
\hline Urinary infections & 250 & $10.6 \%$ \\
\hline Urinary incontinence & 1272 & $54 \%$ \\
\hline $\mathrm{DK}^{\star}$ & 88 & $3.7 \%$ \\
\hline \multicolumn{3}{|l|}{ How does menopause affect sexuality? } \\
\hline No effects & 306 & $13.0 \%$ \\
\hline Decreased sexual desire & 1530 & $65.0 \%$ \\
\hline Decreased frequency of sexual activity & 646 & $27.4 \%$ \\
\hline $\mathrm{DK}^{\star}$ & 186 & $7.9 \%$ \\
\hline \multicolumn{3}{|c|}{ Which of these treatments do you know of for improving vaginal atrophy? } \\
\hline Vaginal lubricants & 1640 & $69.6 \%$ \\
\hline Vibrators & 108 & $4.6 \%$ \\
\hline Laser therapy & 73 & $3.1 \%$ \\
\hline Hydrating Creams & 558 & $23.7 \%$ \\
\hline *DK = Don't know & & \\
\hline
\end{tabular}




\begin{tabular}{|lll|}
\hline & $\mathbf{n}$ & \% of total respondents $(\mathbf{n}=\mathbf{2 3 5 5})$ \\
\hline Estrogen creams & 605 & $25.7 \%$ \\
\hline Sexual intercourse & 353 & $15.0 \%$ \\
\hline Hyaluronic acid & 172 & $7.3 \%$ \\
\hline DK* & 443 & $18.8 \%$ \\
\hline Do you talk to your gynecologist about sexuality? & & \\
\hline Yes & 703 & $29.9 \%$ \\
\hline No & 1564 & $66.4 \%$ \\
\hline DK* & 87 & $3.7 \%$ \\
\hline If no, indicate the reason(s): & \\
\hline Mistrust & 49 & $2.1 \%$ \\
\hline Embarrassment & 254 & $10.8 \%$ \\
\hline He/she has never asked me & 893 & $57.1 \%$ \\
\hline Lack of consultation time & 286 & $18.2 \%$ \\
\hline I do not attend this type of clinic & 374 & $24.0 \%$ \\
\hline I do not think it is important to him/her & 214 & $9.1 \%$ \\
\hline *DK = Don't know & & \\
\hline
\end{tabular}

Among the women surveyed, the most widely known treatments for GSM were lubricants $(69.6 \%)$ followed by local estrogens (25.7\%), whilst $18.8 \%$ of the women did not know of any treatment. The benefit of maintaining sexual activity for vaginal atrophy was recognized by $15 \%$ of women. A total of $66 \%$ of the women surveyed had not discussed the issue of sexuality with their gynecologist, and for most of these women (57.1\%) this was because their gynecologist had never asked them (Table 1).

During the coronavirus confinement period, 204 women with treatment for GSM were asked; The median age was 58 years (IQR 54-60) and the age of menopause 50 years (IQR 49-52). The demographic characteristics of the adherence extension phase and the treatments indicated for GSM in the year prior to the declaration of the state of alarm are shown in Table 2. Poor compliance with treatment was observed in $72.5 \%$ of the patients included in the study. The most frequent causes of poor adherence were omission (67.7\%) and changes in sexual activity (49\%). Less sexual activity during the state of alarm and having been diagnosed with coronavirus infection ( $11.8 \%$ of the women in the study) were significantly associated with poorer treatment compliance $(p<0.001 p=0.003$ respectively) (Table 3). 
Table 2

Clinical and demographic characteristics of the sample analyzed in the extension study of GSM treatment adherence during COVID-19 confinement

\begin{tabular}{|c|c|c|}
\hline & & $\begin{array}{l}n(\%) \\
(n=204)\end{array}$ \\
\hline Age & Median (RIQ*) & $\begin{array}{l}58(54- \\
60)\end{array}$ \\
\hline Years of menopause & Median (RIQ*) & $\begin{array}{l}50(49- \\
52)\end{array}$ \\
\hline \multirow[t]{2}{*}{ Sexually active } & Yes & $176(86.3)$ \\
\hline & No & $28(13.7)$ \\
\hline \multirow[t]{2}{*}{ Level of education } & Basic-medium & $112(54.9)$ \\
\hline & University & $92(45.1)$ \\
\hline \multirow{3}{*}{$\begin{array}{l}\text { Sexual activity during } \\
\text { confinement }\end{array}$} & Decrease & $128(62.7)$ \\
\hline & Stable & $66(32.3)$ \\
\hline & Increase & $10(4.9)$ \\
\hline \multirow[t]{5}{*}{ Indicated treatments } & Lubricants, moisturizers & $48(23.5)$ \\
\hline & Local estrogens & $120(58.8)$ \\
\hline & Prasterone & $20(9.8)$ \\
\hline & Ospemifene & $12(5.9)$ \\
\hline & $\mathrm{MHT}^{\dagger}$ & $4(2)$ \\
\hline \multirow[t]{4}{*}{ Compliance $^{¥}$} & Do you ever forget to take your medication? & $52(25.5)$ \\
\hline & $\begin{array}{l}\text { Do you take the medication at the prescribed } \\
\text { times? }\end{array}$ & $96(47.1)$ \\
\hline & If you feel well, do you not take it? & $52(25.5)$ \\
\hline & If it makes you feel worse, do you not take it? & $4(2)$ \\
\hline
\end{tabular}

¥ Questions included in the Morinsky-Green-Levine test (8).

$\S$ Compliance was considered to be good if the 4 questions were answered as follows: No/Yes/No/No.

${ }^{*}$ RIQ: Interquartile range

${ }^{\dagger} \mathrm{MTH}$ : Menopause hormone therapy 


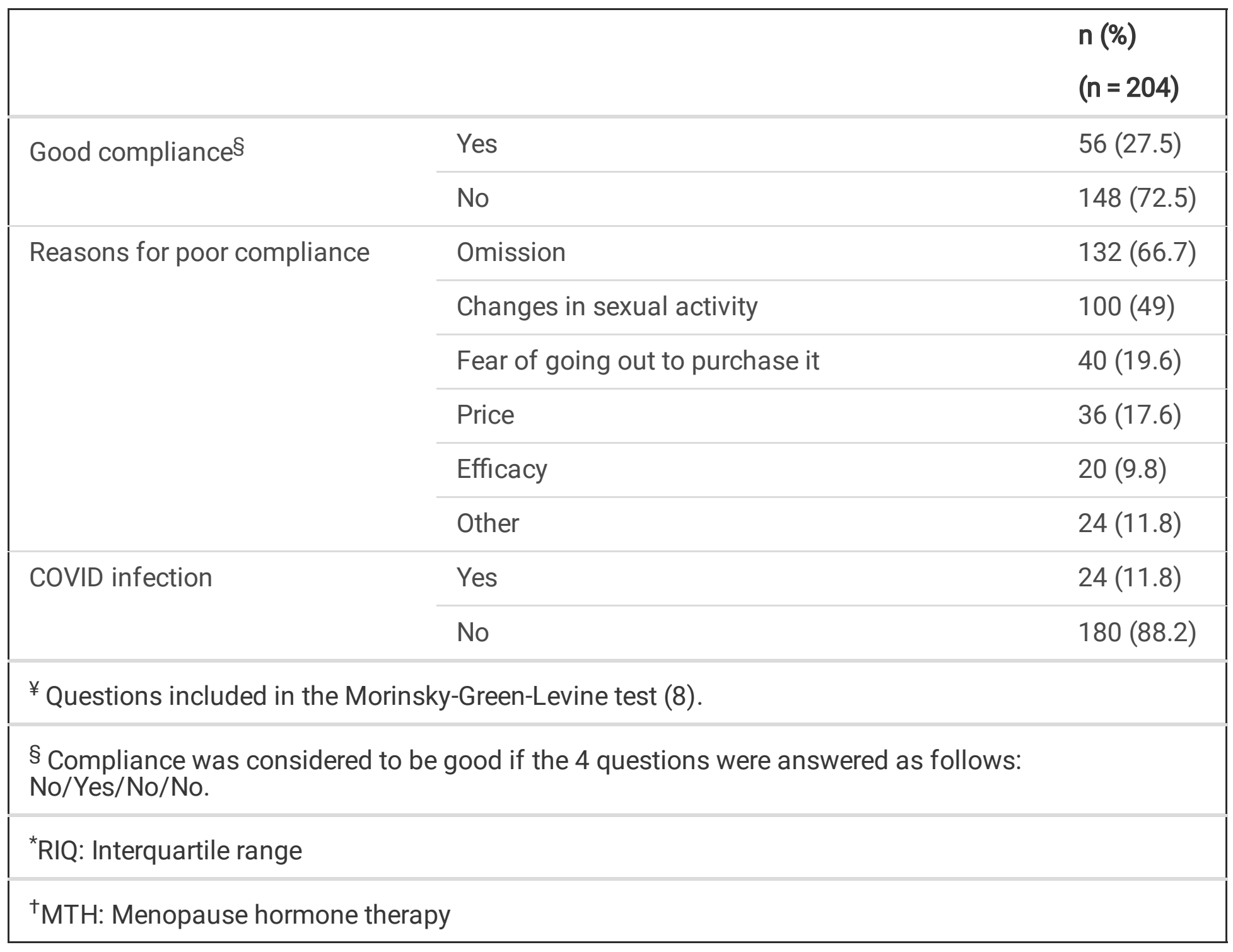


Table 3

Factors related to GSM treatment adherence during COVID-19 confinement

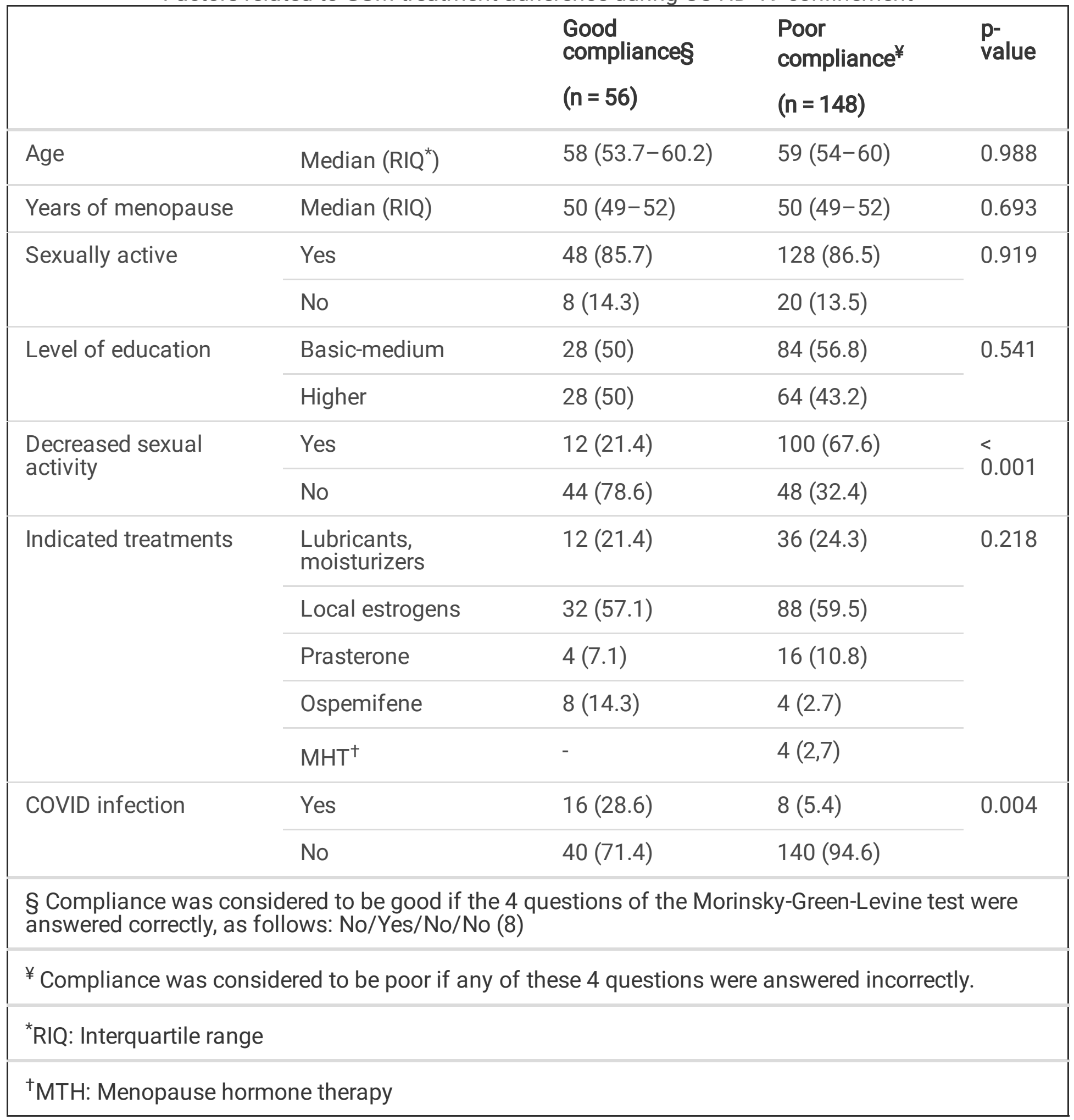

\section{Discussion}

The main finding of this survey is that the sample of women analyzed have poor knowledge about GSM, particularly about treatment options. In addition, there is also a clear lack of communication between women and their gynecologist in relation to the issue of sexuality. To date, this is the largest sample of 
women to have been surveyed about their beliefs and knowledge regarding GSM, its treatments, and associated sexuality issues in Spain. In addition, during the extension phase, we have verified that adherence to GSM treatments has been remarkably poor during the coronavirus confinement period. To our knowledge, this is the first study to assess compliance with treatment for GSM during the coronavirus pandemic.

Several studies have analyzed the prevalence and impact of vulvo-vaginal atrophy (VVA) symptoms experienced during menopause (9-12) The most common symptom among all of these is vaginal dryness, with the European EVES study reporting an estimated prevalence of up to $90 \%$. Dyspareunia is less prevalent $(44-72 \%)$ but is the most bothersome symptom $(9,10,12)$. Sexuality is the most affected area, as indicated by the VIVA (65\%) (11), and the American REVIVE (59\%) surveys (9). In the CLOSER study, the reduction of sexual satisfaction and dyspareunia are causes of significant sexual dysfunction (13). However, in some of these studies, vaginal atrophy was not recognized as a medical condition by several women, who felt that their concerns had been dismissed as a normal part of aging $(9,10,14)$.

When asked about the conditions or symptoms associated with menopause, most of the women in our survey identified vaginal dryness (74.3\%); whilst other conditions such as decreased desire or sexual dysfunction were less recognized ( $44.8 \%$ and $29 \%$ respectively). The attitudes of the women towards their symptoms are highly variable and may depend on sociocultural and even personality factors $(8,9,13)$. For instance, according to the REVIVE surveys $(9,10)$, women in Europe appear to be more aware of the fact that vaginal dryness and dyspareunia are menopausal symptoms $(53 \%)$ than women in the USA $(38 \%)$.

Another factor that could be associated with the perception of VVA symptoms is communication with the clinicians and the attitude of women in relation to raising the issue of GSM, that is, whether they discuss the symptoms with their health care practitioner during their gynecological appointments. When we specifically asked about sexuality in menopause, only a limited number of women believed that menopause does not affect sexuality (13\%). The majority (65\%) thought that menopause decreased sexual desire and almost half (34.9\%) recognized the symptom of pain during sexual intercourse. According to the AGATA study, $78.7 \%$ of 913 patients with VVA had never been questioned by a health care practitioner (15).

In accordance with our findings, the Women's EMPOWER survey, an internet-based survey of US women with VVA symptoms (4), reported that most women were aware of over-the-counter treatments (vaginal lubricants or moisturizers) but knew rather less about options such as local estrogen treatments. Other surveys evaluated the knowledge, behavior, and attitudes associated with GSM; the authors valued treatments women receive, their satisfaction and concerns, but the treatment options that women are aware of are not discussed in depth $(9,10,15,16)$. This is an important aspect to consider if patients are to choose the most appropriate treatment according to their preferences. It is clear that as gynecologists we must improve the information that we provide to our patients in this regard. 
The issue of communication with the clinician in relation to sexuality is particularly interesting. Most of the women in our sample (66.4\%) reported that they did not discuss the topic of sexuality with their gynecologist, the reason for which, according to the majority of women $(57.1 \%)$, was that their clinician had never asked them. In accord with this finding, other studies have shown that women expect their gynecologist to begin the conversation in this regard, and that these women have a strong desire to obtain accurate medical information about VVA (4). A study was conducted in Spain to evaluate whether actively addressing sexuality in a gynecological consultation with postmenopausal patients improves the diagnosis of sexual problems (18). A total of 256 postmenopausal women participated in the study. Of these, $12.1 \%$ reported a sexual problem during the first 5 minutes of the interview. The prevalence of patients with a sexual problem increased by $35.9 \%$ (from 12.1-48.0\%) when they were asked about sexuality after $5 \mathrm{~min}(\mathrm{p}<0.0001)$. These findings suggest that gynecologists should reflect on this issue and consider asking all menopausal women about sexuality.

The clinical efficacy of a treatment over time varies depending on persistence and compliance. Lack of adherence to treatment in chronic diseases is a major problem worldwide (19). In GSM, both adherence and persistence are low. Among women with GSM who participated in the REVIVE study in Spain, 40\% reported having discontinued treatment for GSM (20). However, our data are even more discouraging. To date, no other study has assessed adherence to GSM treatments during the current coronavirus pandemic. These novel findings should encourage clinicians to reflect on the importance of motivating their patients and emphasizing the benefits of good treatment compliance to avoid unfavorable clinical and economic consequences.

One limitation of our study was the possible selection bias. To minimize this source of bias, the size of the sample was increased and participation in the survey was offered to all women regardless of the reason for their medical visit. Nonetheless, we must assume that the obtained conclusions cannot be extrapolated to the general population and are instead limited to the sample included in the study. Nonetheless, this work might be considered a useful exploratory study that could form the basis for future investigations in the general population. Another possible limitation of our study is related to the choice of questionnaire. There are currently no questionnaires in Spanish that have been sufficiently recognized and validated as an instrument for measuring women's knowledge of menopause. Finally, conducting the Morisky-Green-Levine questionnaire by telephone (which was deemed necessary due to the confinement situation) could also be viewed as a limitation, since the obtained data may be rather different to those that would have been yielded by a self-administered test.

\section{Conclusions}

There is a considerable lack of knowledge regarding the treatments used for GSM. Moreover, most women do not talk to their gynecologist about sexuality issues. Adherence to GSM treatments during the coronavirus confinement has been remarkably low. It is therefore of crucial importance to implement educational and motivational programs and to improve clinician-patient communication about GSM and sexuality during menopause. 


\section{Abbreviations}

MHT: menopause hormone therapy

COMEM: "COnocimiento de las Mujeres Españolas en Menopausia", in its Spanish acronym

GSM: Genitourinary syndrome of menopause

IQR: interquartile range

VVA: vulvo-vaginal atrophy

\section{Declarations}

\section{Ethics approval and consent to participate}

The study was approved by the Research Ethics Committees of Aragon, Spain (C.I. PI18/374) and confirmed by each participating Center.

Verbal informed consent was obtained from all participants. Due to the completely anonymous nature of the study, the Ethics Committee approved this procedure. An adittional information document was administered to all participants.

The research was conducted in accordance with Good Clinical Practice standards and the current revision of the Declaration of Helsinki

\section{Consent for publication}

Not applicable. There are no images or details on individuals reported within the manuscript

\section{Availability of data and materials}

The datasets analysed during the current study available from the corresponding author on request.

\section{Competing interests}

The authors declare no conflict of interest

\section{Funding}

The research team received no financial support, participant compensation or funding. 


\section{Authors' contributions}

LB: is one of the study designers, has participated in the elaboration and writing of the article and is the current corresponding author. SS: has carried out data collection in in the treatment adherence phase during the covid-19 pandemic. DP and MH: have carried out data collection NM: is one of the study designers, has participated on the conceptualization and writing of the manuscript and critical revision of it.

All authors read and approved the final manuscript

\section{Acknowledgements}

The surveys have been carried out by the COMEM Study Spanish investigators in the following hospitals: Hospital Universitario Miguel Servet of Zaragoza, Hospital San Jorge of Huesca, Hospital Universitario La Zarzuela of Madrid, Hospital San Carlos of San Fernando, Hospital Insular-Materno Infantil of Las Palmas, Hospital Quirón Salud of Zaragoza , Hospital Clínico San Carlos of Madrid, Hospital Universitario of Castellón, Complejo Hospitalario of Jaén, Hospital Universitario Central of Asturias, Clínica Diatros of Barcelona, Instituto Palacios of Madrid and European Institute of Sexology, Marbella.

The team of seven menopause experts who made the survey was made up of: $L$ Baquedano, $P$ Coronado, N Mendoza, I Ramirez, E de la Viuda, B Otero and S Sánchez.

\section{References}

1. Palacios S, Castelo-Branco $\mathrm{C}$, Currie $\mathrm{H}$, et al. Update on management of genitourinary syndrome of menopause: A practical guide. Maturitas. 2015;82(3):308-313.

2. Cain, V.S.; Johannes, C.B.; Avis, N.E.; Mohr, B.; Schocken, M.; Skurnick, J.; Ory, M. Sexual functioning and practices in a multi-ethnic study of midlife women: Baseline results from SWAN. J. Sex. Res. $2003,40,266-276$.

3. Damsted Petersen, C. Female Sexual Function in Midlife in Kirana. The EFS and ESSM Syllabus of Clinical Sexology, 1st ed.; Medix Publishers: Amsterdam, The Netherlands, 2013; pp. 1173-1197.

4. Krychman M, Graham S, Bernick B, Mirkin S, Kingsberg SA. The Women's EMPOWER Survey: Women's Knowledge and Awareness of Treatment Options for Vulvar and Vaginal Atrophy Remains Inadequate. J Sex Med. 2017 Mar;14(3):425-433

5. Baquedano L, Sánchez S, Aznar T, Cancelo MJ, Escribano JJ, González S, Iglesias E, Jaimes J, Manubens M, Puy J, Mendoza N, Sánchez-Borrego R, Palacios S. Síndrome genitourinario de la menopausia, MenoGuía AEEM. Primera edición 2020

6. Palacios S, Nappi RE, Shapiro M, de Melo NR, Wender MCO, Fernandes CE, Pardini D, Machado RB, Kulak J Jr, Celis-González C, Martino MT, Pizzi RR, Villaseca P, Storch E, Danckers LF, Hernández- 
Bueno JA. An individualized approach to the management of vaginal atrophy in Latin America. Menopause. 2019 Aug;26(8):919-928

7. Baquedano L, Espiau A, Fasero M, Ortega S, Ramirez I, Mendoza N; COMEM Study Spanish investigators. Beliefs, knowledge and the impact of COVID19 on menopause therapies in Spanish women: COMEM-treatment study. BMC Womens Health. 2020 Dec 28;20(1):2771

8. Morisky DE, Green LW, Levine DM. Concurrent and predictive validity of a self-reported measure of medication adherence. Med Care. 1986 Jan;24(1):67-74.

9. Kingsberg SA, Wysocki S, Magnus L, Krychman ML. Vulvar and vaginal atrophy in postmenopausal women: findings from the REVIVE (REal Women's Vlews of Treatment Options for Menopausal Vaginal ChangEs) survey. J Sex Med 2013;10:1790-9.

10. Nappi RE, Palacios S, Panay N, Particco M, Krychman ML.Vulvar and vaginal atrophy in four European countries: evidence from the European REVIVE Survey. Climacteric 2015;1-10.

11. Nappi RE, Kokot-Kierepa M. Vaginal Health: Insights, Views \& Attitudes (VIVA) - results from an international survey. Climacteric 2012;15:36-44.

12. Palacios S, Nappi RE, Bruyniks N, Particco M, Panay N. The European Vulvovaginal Epidemiological Survey (EVES): prevalence, symptoms and impact of vulvovaginal atrophy of menopause. Climacteric. 2018 Mar 19;21(3):286-91

13. Nappi RE, Mattsson LA, Lachowsky M, Maamari R, Giraldi A. The CLOSER sur- vey: impact of postmenopausal vaginal discomfort on relationships between women and their partners in Northern and Southern Europe. Maturitas 2013;75:373-9.

14. Utian WH, Maamari R. Attitudes and approaches to vaginal atrophy in postmenopausal women: a focus group qualitative study. Climacteric. 2014;17(1):29-36

15. Palma F, Della Vecchia E, Cagnacci A; as the Writing Group of the AGATA study. Medical and patient attitude towards vaginal atrophy: the AGATA study. Climacteric. 2016;19(6):553-557.

16. Simon JA, Kokot-Kierepa M, Goldstein J, et al. Vaginal health in the United States: results from the Vaginal Health: Insights, Views \& Attitudes survey. Menopause 2013;20:1043-1048.

17. Harun MGD, Salema U, Chowdhury A, Haque MI, Kafi MAH, Shahajahan M, Sharmin S. Knowledge and attitudes associated with menopause among women aged 45 to 60 years: a pilot study among rural and urban women in Bangladesh. Menopause. 2020 Jun;27(6):648-657.

18. Cuerva MJ, Gonzalez D, Canals M, et al. The sexual health approach in postmenopause: The fiveminutes study. Maturitas. 2018;108:31-36.

19. Sabaté E, editor. World Health Organization. Adherence to Long-Term Therapies: Evidence for Action. Geneva, Switzerland: World Health Organization; 2003

20. Palacios S, Cancelo MJ, Castelo Branco C, Llaneza P, Molero F, Borrego RS. Vulvar and vaginal atrophy as viewed by the Spanish REVIVE participants: symptoms, management and treatment perceptions. Climacteric. 2017 Jan 2;20(1):55-61 